\title{
On Reachability in Parameterized Phaser Programs
}

\author{
Zeinab Ganjei ${ }^{1}$, Ahmed Rezine ${ }^{1(凶)}$, Ludovic Henrio ${ }^{2}$, Petru Eles ${ }^{1}$, \\ and Zebo Peng ${ }^{1}$ \\ 1 Linköping University, Linköping, Sweden \\ \{zeinab.ganjei, ahmed.rezine, petru.eles, zebo.peng\}@liu.se \\ 2 Univ Lyon, EnsL, UCBL, CNRS, Inria, LIP, 69342 Lyon Cedex 07, France \\ ludovic.henrio@ens-lyon.fr
}

\begin{abstract}
We address the problem of statically checking safety properties (such as assertions or deadlocks) for parameterized phaser programs. Phasers embody a non-trivial and modern synchronization construct used to orchestrate executions of parallel tasks. This generic construct supports dynamic parallelism with runtime registrations and deregistrations of spawned tasks. It generalizes many synchronization patterns such as collective and point-to-point schemes. For instance, phasers can enforce barriers or producer-consumer synchronization patterns among all or subsets of the running tasks. We consider in this work programs that may generate arbitrarily many tasks and phasers. We propose an exact procedure that is guaranteed to terminate even in the presence of unbounded phases and arbitrarily many spawned tasks. In addition, we prove undecidability results for several problems on which our procedure cannot be guaranteed to terminate.
\end{abstract}

\section{Introduction}

We focus on the parameterized verification problem of parallel programs that adopt the phasers construct for synchronization [15]. This coordination construct unifies collective and point-to-point synchronization. Parameterized verification is particularly relevant for mainstream parallel programs as the number of interdependent tasks in many applications, from scientific computing to web services or e-banking, may not be known apriori. Parameterized verification of phaser programs is a challenging problem due to the arbitrary numbers of involved tasks and phasers. In this work, we address this problem and provide an exact symbolic verification procedure. We identify parameterized problems for which our procedure is guaranteed to terminate and prove the undecidability of several variants on which our procedure cannot be guaranteed to terminate in general.

Phasers build on the clock construct from the X10 programming language [5] and are implemented in Habanero Java [4]. They can be added to any parallel programming language with a shared address space. Conceptually, phasers are synchronization entities to which tasks can be registered or unregistered. 
Registered tasks may act as producers, consumers, or both. Tasks can individually issue signal, wait, and next commands to a phaser they are registered to. Intuitively, a signal command is used to inform other tasks registered to the same phaser that the issuing task is done with its current phase. It increments the signal value associated to the issuing task on the given phaser. The wait command on the other hand checks whether all signal values in the phaser are strictly larger than the number of waits issued by this task, i.e. all registered tasks have passed the issuing task's wait phase. It then increments the wait value associated to the task on the phaser. As a result, the wait command might block the issuing task until other tasks issue enough signals. The next command consists in a signal followed by a wait. The next command may be associated to a sequence of statements that are to be executed in isolation by one of the registered tasks participating in the command. A program that does not use this feature of the next statement is said to be non-atomic. A task deregisters from a phaser by issuing a drop command on it.

The dynamic synchronization allowed by the construct suits applications that need dynamic load balancing (e.g., for solving non-uniform problems with unpredictable load estimates [17]). Dynamic behavior is enabled by the possible runtime creation of tasks and phasers and their registration/de-registration. Moreover, the spawned tasks can work in different phases, adding flexibility to the synchronization pattern. The generality of the construct makes it also interesting from a theoretical perspective, as many language constructs can be expressed using phasers. For example, synchronization barriers of Single Program Multiple Data programs, the Bulk Synchronous Parallel computation model [16], or promises and futures constructs [3] can be expressed using phasers.

We believe this paper provides general (un)decidability results that will guide verification of other synchronization constructs. We identify combinations of features (e.g., unbounded differences between signal and wait phases, atomic statements) and properties to be checked (e.g., assertions, deadlocks) for which the parameterized verification problem becomes undecidable. These help identify synchronization constructs with enough expressivity to result in undecidable parameterized verification problems. We also provide a symbolic verification procedure that terminates even on fragments with arbitrary phases and numbers of spawned tasks. We get back to possible implications in the conclusion:

- We show an operational model for phaser programs based on $[4,6,9,15]$.

- We propose an exact symbolic verification procedure for checking reachability of sets of configurations for non-atomic phaser programs even when arbitrarily many tasks and phasers may be generated.

- We prove undecidability results for several reachability problems.

- We show termination of our procedure when checking assertions for nonatomic programs even when arbitrary many tasks may be spawned.

- We show termination of our procedure when checking deadlock-freedom and assertions for non-atomic programs with bounded gaps between signal and wait values, even when arbitrary many tasks may be spawned. 
results in the task incrementing its signal phase associated to the phaser. This is non-blocking. On the other-hand, issuing a wait command by a task on a phaser $p$ will block until all tasks registered to $p$ get signal values on $p$ that are strictly larger than the wait value of the issuing task. The wait phase of the issuing task is then incremented. Intuitively, signals allow issuing tasks to state other tasks need not wait for them. In retrospect, waits allow tasks to make sure all registered tasks have moved past their wait phases.

Upon creation of a phaser, wait and signal phases are initialized to 0 (except in WAIT mode where no signal phase is associated to the task in order to not block other waiters). The only other way a task may get registered to a phaser is if an already registered task spawns and registers it in the same mode (or in WAIT or SIg if the registrar is registered in SIG_WAIT). In this case, wait and signal phases of the newly registered task are initialized to those of the registrar. Tasks are therefore dynamically registered (e.g., lines 7-8). They can also dynamically deregister themselves (e.g., line 10-11).

Here, an unbounded number of producers and consumers synchronize using two phasers. Consumers require producers to be ahead wrt. the phaser they point to with p. At the same time, consumers need to be ahead of all producers wrt. the phaser pointed to with c. It should be clear that phasers can be used as barriers for synchronizing dynamic subsets of concurrent tasks. Observe that tasks need not, in general, proceed in a lock step fashion. The difference between the largest signal value and the smallest wait value can be arbitrarily large (several signals before waits catch up). This allows for more flexibility.

We are interested in checking: (a) control reachability as in assertions (e.g., line 20), race conditions (e.g., mutual exclusion of lines 20 and 33) or registration errors (e.g., signaling a dropped phaser), and (b) plain reachability as in deadlocks (e.g., a producer at line 19 and a consumer at line 30 with equal phases waiting for each other). Both problems deal with reachability of sets of configurations. The difference is that control state reachability defines the targets with the states of the tasks (their control locations and whether they are registered to some phasers). Plain reachability can, in addition, constrain values of the phases in the target configurations (e.g., requiring equality between wait and signal values for deadlocks).

\section{Phaser Programs and Reachability}

We describe syntax and semantics of a core language. We make sure the language is representative of general purpose languages with phasers so that our results have a practical impact. A phaser program prg $=(\mathrm{B}, \mathrm{V}, \mathrm{T})$ involves a set $\mathrm{T}$ of tasks including a unique "main" task main ()$\{$ stmt $\}$. Arbitrary many instances of each task might be spawned during a program execution. All task instances share a set $B$ of Boolean variables and make use of a set $\mathrm{V}$ of phaser variables that are local to individual task instances. Arbitrary many phasers might also be generated during program execution. Syntax of programs is as follows. 


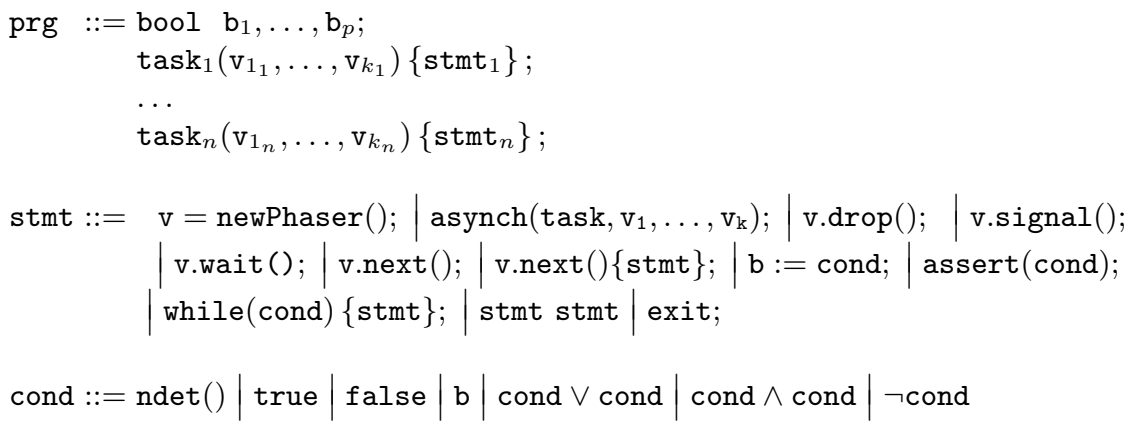

Initially, a unique task instance starts executing the main ()$\{$ stmt $\}$ task. A phaser can recall a pair of values (i.e., wait and signal) for each task instance registered to it. A task instance can create a new phaser with $\mathrm{v}=$ newPhaser () , get registered to it (i.e., gets zero as wait and signal values associated to the new phaser) and refer to the phaser with its local variable v. We simplify the presentation by assuming all registrations to be in SIG_WAIT mode. Including the other modes is a matter of depriving WAIT-registered tasks of a signal value (to ensure they do not block other registered tasks) and of ensuring issued commands respect registration modes. We use $\mathrm{V}$ for the union of all local phaser variables. A task task $\left(\mathrm{v}_{1}, \ldots, \mathrm{v}_{k}\right)\{$ stmt $\}$ in $\mathrm{T}$ takes the phaser variables $\mathrm{v}_{1}, \ldots \mathrm{v}_{k}$ as parameters (write param0f(task) to mean these parameters). A task instance can spawn another task instance with asynch(task, $\left.\mathrm{v}_{1}, \ldots, \mathrm{v}_{\mathrm{n}}\right)$. The issuing task instance registers the spawned task to the phasers pointed to by $\mathrm{v}_{1}, \ldots, \mathrm{v}_{n}$, with its own wait and signal values. Spawner and Spawnee execute concurrently. A task instance can deregister itself from a phaser with v.drop().

A task instance can issue signal or wait commands on a phaser referenced by $\mathrm{v}$ and on which it is registered. A wait command on a phaser blocks until the wait value of the task instance executing the wait on the phaser is strictly smaller than the signal value of all task instances registered to the phaser. In other words, v.wait () blocks if $\mathrm{v}$ points to a phaser such that at least one of the signal values stored by the phaser is equal to the wait value of the task that tries to perform the wait. A signal command does not block. It only increments the signal value of the task instance executing the signal command on the phaser. v.next () is syntactic sugar for a signal followed by a wait. Moreover, v.next ()$\{$ stmt $\}$ is similar to v.next() but the block of code stmt is executed atomically by exactly one of the tasks participating in the synchronization before all tasks continue the execution that follows the barrier. v.next ()$\{$ stmt $\}$ thus requires all tasks to be synchronized on exactly the same statement and is less flexible. Absence of a v.next ()$\{$ stmt $\}$ makes a program non-atomic.

Note that assignment of phaser variables is excluded from the syntax; additionally, we restrict task creation $\operatorname{asynch}\left(\operatorname{task}, \mathrm{v}_{1}, \ldots, \mathrm{v}_{\mathrm{n}}\right)$ and require that parameter variables $\mathrm{v}_{i}$ are all different. This prevents two variables from pointing to the same phaser and avoids the need to deal with aliasing: we can reason 
on the single variable in a process that points to a phaser. Extending our work to deal with aliasing is easy but would require heavier notations.

We will need the notions of configurations, partial configurations and inclusion in order to define the reachability problems we consider in this work. We introduce them in the following and assume a phaser program prg $=(\mathrm{B}, \mathrm{V}, \mathrm{T})$.

Configurations. Configurations of a phaser program describe valuations of its variables, control sequences of its tasks and registration details to the phasers.

Control sequences. We define the set Suff of control sequences of prg to be the set of suffixes of all sequences stmt appearing in some statement task $(\ldots)\{$ stmt $\}$. In addition, we define UnrSuff to be the smallest set containing Suff in addition to the suffixes of all (i) $\mathrm{s}_{1}$; while(cond) $\left\{\mathrm{s}_{1}\right\} ; \mathrm{s}_{2}$ if while(cond) $\left\{\mathrm{s}_{1}\right\} ; \mathrm{s}_{2}$ is in UnrSuff, and of all (ii) $\mathbf{s}_{1} ; \mathbf{s}_{2}$ if $\operatorname{if}(\mathrm{cond})\left\{\mathbf{s}_{1}\right\} ; \mathbf{s}_{2}$ is in UnrSuff, and of all (iii) $\mathbf{s}_{1} ; \operatorname{v.next}()\{\} ; \mathbf{s}_{2}$ if v.next ()$\left\{\mathbf{s}_{1}\right\} ; \mathbf{s}_{2}$ in UnrSuff, and finally of all (iv) v.signal(); v.wait(); $\mathbf{s}_{2}$ if v.next() \{\}$; \mathbf{s}_{2}$ is in UnrSuff. We write hd(s) and $\mathrm{tl}(\mathrm{s})$ to respectively mean the head and the tail of a sequence $\mathrm{s}$.

Partial configurations. Partial configurations allow the characterization of sets of configurations by partially stating some of their common characteristics. A partial configuration $c$ of $\operatorname{prg}=(\mathrm{B}, \mathrm{V}, \mathrm{T})$ is a tuple $(\mathcal{T}, \mathcal{P}, 6 v$, seq, phase $)$ where:

- $\mathcal{T}$ is a finite set of task identifiers. We let $t, u$ range over the values in $\mathcal{T}$.

$-\mathcal{P}$ is a finite set of phaser identifiers. We let $p, q$ range over the values in $\mathcal{P}$.

- $6 v: \mathrm{B} \rightarrow \mathbb{B}^{\{*\}}$ fixes the values of some of the shared variables. ${ }^{1}$

- seq $: \mathcal{T} \rightarrow$ UnrSuff ${ }^{\{*\}}$ fixes the control sequences of some of the tasks.

- phase $: \mathcal{T} \rightarrow$ partialfunctions $\left(\mathcal{P}, \mathrm{V}^{\{-, *\}} \times\left(\mathbb{N}^{2} \cup\{(*, *), \mathrm{nreg}\}\right)\right)$ is a mapping that associates to each task $t$ in $\mathcal{T}$ a partial mapping stating which phasers are known by the task and with which registration values.

Intuitively, partial configurations are used to state some facts about the valuations of variables and the control sequences of tasks and their registrations. Partial configurations leave some details unconstrained using partial mappings or the symbol $*$. For instance, if $6 v(\mathrm{~b})=*$ in a partial configuration $(\mathcal{T}, \mathcal{P}, 6 v$, seq, phase $)$, then the partial configuration does not constrain the value of the shared variable $\mathrm{b}$. Moreover, a partial configuration does not constrain the relation between a task $t$ and a phaser $p$ when phase $(t)(p)$ is undefined. Instead, when the partial mapping phase $(t)$ is defined on phaser $p$, it associates a pair phase $(t)(p)=\left(\right.$ var, val) to $p$. If $\operatorname{var} \in \mathrm{V}^{\{-, *\}}$ is a variable $\mathrm{v} \in \mathrm{V}$ then the task $t$ in $\mathcal{T}$ uses its variable $\mathrm{v}$ to refer to the phaser $p$ in $\mathcal{P}^{2}$. If var is the symbol - then the task $t$ does not refer to $\mathrm{v}$ with any of its variables in $\mathrm{V}$. If var is the symbol $*$, then the task might or might not refer to $p$. The value val in phase $(t)(p)=($ var, val) is either the value nreg or a pair $(w, s)$. The value nreg means the task $t$ is not registered to phaser $p$. The pair $(w, s)$ belongs to $(\mathbb{N} \times \mathbb{N}) \cup\{(*, *)\}$. In this case, task $t$ is registered to phaser $p$ with a wait phase

\footnotetext{
${ }^{1}$ For any set $S, S^{\{a, b, \ldots\}}$ denotes $S \cup\{a, b, \ldots\}$.

2 The uniqueness of this variable is due to the absence of aliasing discussed above.
} 


$$
\frac{\operatorname{hd}(\operatorname{seq}(t))=\operatorname{assert}(\text { cond }) \llbracket \text { cond } \rrbracket_{b v}=\text { false }}{(\mathcal{T}, P, b v, \text { seq, phase }) \in \text { AssertErrors }} \text { (assertion) }
$$

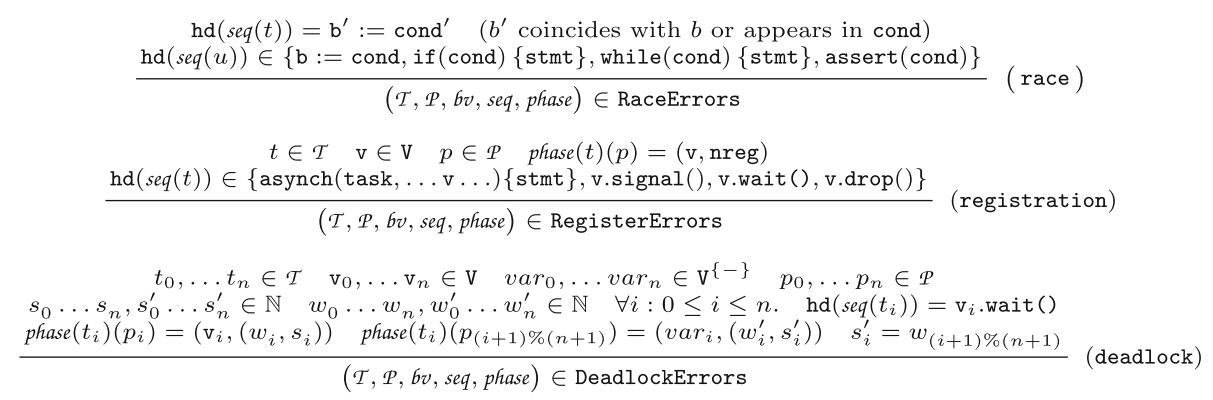

Fig. 2. Definition of error configurations. For instance, a deadlock is obtained in $(\mathcal{T}, \mathcal{P}, 6 v$, seq, phase $)$ if tasks $\left\{t_{0}, \ldots, t_{n}\right\} \subseteq \mathcal{T}$ form a cycle where each $t_{i}$ blocks the wait being executed by $t_{(i+1) \%(n+1)}$ on phaser $p_{(i+1) \%(n+1)}$.

$w$ and a signal phase $s$. The value $*$ means that the wait phase $w$ (resp. signal phase $s$ ) can be any value in $\mathbb{N}$. For instance, phase $(t)(p)=(\mathrm{v}, \mathrm{nreg})$ means variable $\mathrm{v}$ of the task $t$ refers to phaser $p$ but the task is not registered to $p$. On the other hand, phase $(t)(p)=(-,(*, *))$ means the task $t$ does not refer to $p$ but is registered to it with arbitrary wait and signal phases.

Concrete configurations. A concrete configuration (or configuration for short) is a partial configuration $(\mathcal{T}, \mathcal{P}, 6 v, s e q$, phase) where phase $(t)$ is total for each $t \in \mathcal{T}$ and where the symbol $*$ does not appear in any range. It is a tuple $(\mathcal{T}, \mathcal{P}, 6 v$, seq, phase $)$ where $6 v: \mathrm{B} \rightarrow \mathbb{B}$, seq $: \mathcal{T} \rightarrow$ UnrSuff, and phase : $\mathcal{T} \rightarrow$ totalFunctions $\left(\mathcal{P}, \mathrm{V}^{\{-\}} \times((\mathbb{N} \times \mathbb{N}) \cup\{\mathrm{nreg}\})\right)$. For a concrete configuration $(\mathcal{T}, \mathcal{P}, 6 v$, seq, phase $)$, we write isReg (phase, $t, p)$ to mean the predicate phase $(t)(p) \notin\left(\mathrm{V}^{\{-\}} \times\{\right.$nreg $\left.\}\right)$. The predicate isReg $($phase $, t, p)$ captures whether the task $t$ is registered to phaser $p$ according to the mapping phase.

Inclusion of configurations. A configuration $c^{\prime}=\left(\mathcal{T}^{\prime}, \mathcal{P}^{\prime}, 6 v^{\prime}, s e q^{\prime}\right.$, phase $\left.{ }^{\prime}\right)$ includes a partial configuration $c=(\mathcal{T}, \mathcal{P}, 6 v$, seq, phase $)$ if renaming and deleting tasks and phasers from $c^{\prime}$ can give a configuration that "matches" $c$. More formally, $c^{\prime}$ includes $c$ if $\left(\left(6 v(\mathrm{~b}) \neq 6 v^{\prime}(\mathrm{b})\right) \Longrightarrow(6 v(\mathrm{~b})=*)\right)$ for each $\mathrm{b} \in \mathrm{B}$ and there are injections $\tau: \mathcal{T} \rightarrow \mathcal{T}^{\prime}$ and $\pi: \mathcal{P} \rightarrow \mathcal{P}^{\prime}$ s.t. for each $t \in \mathcal{T}$ and $p \in \mathcal{P}:$ (1) $\left(\left(\operatorname{seq}(t) \neq \operatorname{seq}^{\prime}(\tau(t))\right) \Longrightarrow(\operatorname{seq}(t)=*)\right)$, and either $(2$. a) phase $(t)(p)$ is undefined, or (2.b) phase $(t)(p)=($ var, val $)$ and phase ${ }^{\prime}(\tau(t))(\pi(p))=\left(\right.$ var $\left.^{\prime}, v^{\prime} l^{\prime}\right)$ with $((v a r \neq$ $\left.\left.v a r^{\prime}\right) \Longrightarrow(v a r=*)\right)$ and either $\left(v a l=v a l^{\prime}=\mathrm{nreg}\right)$ or $v a l=(w, s)$ and $v a l^{\prime}=\left(w^{\prime}, s^{\prime}\right)$ with $\left(\left(w \neq w^{\prime}\right) \Longrightarrow(w=*)\right)$ and $\left(\left(s \neq s^{\prime}\right) \Longrightarrow(s=*)\right)$.

Semantics and reachability. Given a program prg $=(B, V, T)$, the main task main ()$\{$ stmt $\}$ starts executing stmt from an initial configuration $c_{\text {init }}=$ $\left(\mathcal{T}_{\text {init }}, \mathcal{P}_{\text {init }}, 6 v_{\text {init }}, \boldsymbol{s e q} q_{\text {init }}\right.$, phase $\left._{\text {init }}\right)$ where $\mathcal{T}_{\text {init }}$ is a singleton, $\mathcal{P}_{\text {init }}$ is empty, $6 v_{\text {init }}$ sends all shared variables to false and seq $_{\text {init }}$ associates stmt to the unique task in $\mathcal{T}_{\text {init }}$. We write $c \underset{\text { stmt }}{\stackrel{t}{\longrightarrow}} c^{\prime}$ to mean a task $t$ in $c$ can fire statement stmt 


$$
\begin{aligned}
& \operatorname{hd}(\operatorname{seq}(t))=\mathrm{v}:=\text { newPhaser }() \quad p \notin \mathcal{P} \quad \mathcal{P}^{\prime}=\mathcal{P} \cup\{p\} \quad \text { phase }^{\prime}=\text { phase }[t \leftarrow\{p \leftarrow(\mathrm{v},(0,0))\}] \\
& \text { phase }^{\prime \prime}=\text { phase }^{\prime}\left[\left\{u \leftarrow \text { phase }^{\prime}(u)[p \leftarrow(-, \text { nreg })] \mid u \in \mathcal{T} \backslash\{t\}\right\}\right] \\
& (\mathcal{T}, P, 6 v, \text { seq, phase }) \underset{\mathrm{v}:=\text { newPhaser }()}{\longrightarrow}\left(\mathcal{T}, \mathcal{P}^{\prime}, b v, \text { seq }[t \leftarrow \operatorname{tl}(\text { seq }(t))], \text { phase }^{\prime \prime}\right)
\end{aligned}
$$

Fig. 3. Operational semantics of phaser statements. Each transition corresponds to a task $t \in \mathcal{T}$ executing a statement from a configuration $(\mathcal{T}, \mathcal{P}, 6 v$, seq, phase $)$. For instance, the drop transition corresponds to a task $t$ executing v.drop () when registered to phaser $p \in \mathcal{P}$ (with phases $(w, s)$ ) and refering to it with variable $\mathrm{v}$. The result is the same configuration where task $t$ moves to its next statement without being registered to $p$.

resulting in configuration $c^{\prime}$. See Fig. 3 for a description of phaser semantics. We write $c \underset{\text { stmt }}{\longrightarrow} c^{\prime}$ if $c \underset{\text { stmt }}{\stackrel{t}{\longrightarrow}} c^{\prime}$ for some task $t$ and $c \rightarrow c^{\prime}$ if $c \underset{\text { stmt }}{\longrightarrow} c^{\prime}$ for some stmt. We write $\underset{\text { stmt }}{+}$ for the transitive closure of $\underset{\text { stmt }}{\longrightarrow}$ and let $\rightarrow$ be the reflexive transitive closure of $\rightarrow$. Figure 2 identifies erroneous configurations.

We are interested in the reachability of sets of configurations (i.e., checking safety properties). We differentiate between two reachability problems depending on whether the target sets of configurations constrain the registration phases or not. The plain reachability problem may constrains the registration phases of the target configurations. The control reachability problem may not. We will see that decidability of the two problems can be different. The two problems are defined in the following.

Plain reachability. First, we define equivalent configurations. A configuration $c=(\mathcal{T}, \mathcal{P}, 6 v$, seq, phase $)$ is equivalent to configuration $c^{\prime}=\left(\mathcal{T}^{\prime}, \mathcal{P}^{\prime}, 6 v^{\prime}\right.$, seq $q^{\prime}$, phase $\left.e^{\prime}\right)$ if $6 v=6 v^{\prime}$ and there are bijections $\tau: \mathcal{T} \rightarrow \mathcal{T}^{\prime}$ and $\pi: \mathcal{P} \rightarrow \mathcal{P}^{\prime}$ such that, for 
all $t \in \mathcal{T}, p \in \mathcal{P}$ and var $\in \mathrm{V}^{\{-\}}$, seq $(t)=\operatorname{seq}^{\prime}(\tau(t))$ and there are some integers $\left(k_{p}\right)_{p \in \mathcal{P}}$ such that phase $(t)(p)=(\operatorname{var},(w, s))$ iff phase $^{\prime}(\tau(t))(\pi(p))=(\operatorname{var},(w+$ $\left.\left.k_{p}, s+k_{p}\right)\right)$. We write $c \sim c^{\prime}$ to mean that $c$ and $c^{\prime}$ are equivalent. Intuitively, equivalent configurations simulate each other. We can establish the following:

Lemma 1 (Equivalence). Assume two configurations $c_{1}$ and $c_{2}$. If $c_{1} \rightarrow c_{2}$ and $c_{1}^{\prime} \sim c_{1}$ then there is a configuration $c_{2}^{\prime}$ s.t. $c_{2}^{\prime} \sim c_{2}$ and $c_{1}^{\prime} \rightarrow c_{2}^{\prime}$.

Observe that if the wait value of a task $t$ on a phaser $p$ is equal to the signal of a task $t^{\prime}$ on the same phaser $p$ in some configuration $c$, then this is also the case, up to a renaming of the phasers and tasks, in all equivalent configurations. This is particularly relevant for defining deadlock configurations where a number of tasks are waiting for each other. The plain reachability problem is given a program and a target partial configuration and asks whether a configuration (equivalent to a configuration) that includes the target partial configuration is reachable.

More formally, given a program prg and a partial configuration $c$, let $c_{\text {init }}$ be the initial configuration of prg, then reach $(\operatorname{prg}, c)$ if and only if $c_{\text {init }} \rightarrow^{*} c_{1}$ for $c_{1} \sim c_{2}$ and $c_{2}$ includes $c$.

Definition 1 (Plain reachability). For a program prg and a partial configuration $c$, decide whether reach $(\mathrm{prg}, c)$ holds.

Control reachability. A partial configuration $c=(\mathcal{T}, \mathcal{P}, 6 v$, seq, phase $)$ is said to be a control partial configuration if for all $t \in \mathcal{T}$ and $p \in \mathcal{P}$, either phase $(t)(p)$ is undefined or phase $(t)(p) \in\left(\mathrm{V}^{\{-, *\}} \times\{(*, *)\right.$, nreg $\left.\}\right)$. Intuitively, control partial configurations do not constrain phase values. They are enough to characterize, for example, configurations where an assertion is violated (see Fig. 2).

Definition 2 (Control reachability). For a program prg and a control partial configuration c, decide whether reach(prg, c) holds.

Observe that plain reachability is at least as hard to answer as control reachability since any control partial configuration is also a partial configuration. It turns out the control reachability problem is undecidable for programs resulting in arbitrarily many tasks and phasers as stated by the theorem below. This is proven by reduction of the state reachability problem for 2-counter Minsky machines. A 2-counter Minsky machine $\left(S,\left\{x_{1}, x_{2}\right\}, \Delta, s_{0}, s_{F}\right)$ has a finite set $S$ of states, two counters $\left\{x_{1}, x_{2}\right\}$ with values in $\mathbb{N}$, an initial state $s_{0}$ and a final state $s_{F}$. Transitions may increment, decrement or test a counter. For example $\left(s_{0}, \operatorname{test}\left(x_{1}\right), s_{F}\right)$ takes the machine from $s_{0}$ to $s_{F}$ if the counter $x_{1}$ is zero.

Theorem 1 (Minsky machines [14]). Checking whether $s_{F}$ is reachable from configuration $\left(s_{0}, 0,0\right)$ for 2-counter machines is undecidable in general.

Theorem 2. Control reachability is undecidable in general.

Proof sketch. State reachability of an arbitrary 2-counters Minsky machine is encoded as the control reachability problem of a phaser program. The phaser 
program (see [10]) has three tasks main, xUnit and yUnit. It uses Boolean shared variables to encode the state $s \in S$ and to pass information between different task instances. The phaser program builds two chains, one with xUnit instances for the $x$-counter, and one with yUnit instances for the $y$-counter. Each chain alternates a phaser and a task and encodes the values of its counter with its length. The idea is to have the phaser program simulate all transitions of the counter machine, i.e., increments, decrements and tests for zero. Answering state reachability of the counter machine amounts to checking whether there are reachable configurations where the boolean variables encoding the counter machine can evaluate to the target machine state $s_{F}$.

\section{A Gap-Based Symbolic Representation}

The symbolic representation we propose builds on the following intuitions. First, observe the language semantics impose, for each phaser, the invariant that signal values are always larger or equal to wait values. We can therefore assume this fact in our symbolic representation. In addition, our reachability problems from Sect. 3 are defined in terms of reachability of equivalence classes, not of individual configurations. This is because configurations violating considered properties (see Fig. 2) are not defined in terms of concrete phase values but rather in terms of relations among them (in addition to the registration status, control sequences and variable values). Finally, we observe that if a wait is enabled with smaller gaps on a given phaser, then it will be enabled with larger ones. We therefore propose to track the gaps of the differences between signal and wait values wrt. to an existentially quantified level (per phaser) that lies between wait and signal values of all tasks registered to the considered phaser.

We formally define our symbolic representation and describe a corresponding entailment relation. We also establish a desirable property (namely being a $\mathcal{W} \mathcal{Q O}$, i.e., well-quasi-ordering $[1,8])$ on some classes of representations. This is crucial for the decidability of certain reachability problems (see Sect.5).

Named gaps. A named gap is associated to a task-phaser pair. It consists in a tuple $($ var, val $)$ in $\mathbb{G}=\left(\mathrm{V}^{\{-, *\}} \times\left(\left(\mathbb{N}^{4} \cup\left(\mathbb{N}^{2} \times\{\infty\}^{2}\right)\right) \cup\{\mathrm{nreg}\}\right)\right)$. Like for partial configurations in Sect.3, var $\in \mathrm{V}^{\{-, *\}}$ constrains variable values. The val value describes task registration to the phaser. If registered, then val is a 4-tuple (1w, Is, uw, us). This intuitively captures, together with some level $l$ common to all tasks registered to the considered phaser, all concrete wait and signal values $(w, s)$ satisfying $l_{\mathrm{w}} \leq(l-w) \leq \mathrm{uw}$ and $\mathrm{ls} \leq(s-l) \leq \mathrm{us}$. A named gap $(\operatorname{var},(1 \mathrm{w}, 1 \mathrm{~s}, \mathrm{uw}, \mathrm{us}))$ is said to be free if uw $=\mathrm{us}=\infty$. It is said to be $B$-gap-bounded, for $B \in \mathbb{N}$, if both uw $\leq B$ and us $\leq B$ hold. A set $\mathcal{G} \subseteq \mathbb{G}$ is said to be free (resp. $B$-gap-bounded) if all its named gaps are free (resp. $B$-gap-bounded). The set $\mathcal{G}$ is said to be $B$-good if each one of its named gaps is either free or $B$-gap-bounded. Finally, $\mathcal{G}$ is said to be $\operatorname{good}$ if it is $B$-good for some $B \in \mathbb{N}$. Given a set $\mathcal{G}$ of named gaps, we define the partial order $\unlhd$ on $\mathcal{G}$, and write $(v a r, v a l) \unlhd\left(v a r^{\prime}, v a l^{\prime}\right)$, to mean (i) $\left(v a r \neq v a r^{\prime} \Rightarrow v a r=*\right)$, and (ii) 
$(v a l=\mathrm{nreg}) \Longleftrightarrow\left(v a l^{\prime}=\mathrm{nreg}\right)$, and (iii) if $v a l=(1 \mathrm{w}, \mathrm{ls}, \mathrm{uw}, \mathrm{us})$ and $v a l^{\prime}=$ $\left(1 \mathrm{w}^{\prime}, 1 \mathrm{~s}^{\prime}, \mathrm{uw}^{\prime}, \mathrm{us}^{\prime}\right)$ then $1 \mathrm{w} \leq 1 \mathrm{w}^{\prime}, 1 \mathrm{~s} \leq 1 \mathrm{~s}^{\prime}, \mathrm{uw}^{\prime} \leq \mathrm{uw}$ and $\mathrm{us}^{\prime} \leq \mathrm{us}$. Intuitively, named gaps are used in the definition of constraints to capture relations (i.e., reference, registration and possible phases) of tasks and phasers. The partial order $(v a r, v a l) \unlhd\left(v a r^{\prime}, v a l^{\prime}\right)$ ensures relations allowed by $\left(v a r^{\prime}, v a l^{\prime}\right)$ are also allowed by $(v a r, v a l)$.

Constraints. A constraint $\phi$ of prg $=(\mathrm{B}, \mathrm{V}, \mathrm{T})$ is a tuple $(\mathcal{T}, \mathcal{P}, 6 v$, seq, gap, egap $)$ that denotes a possibly infinite set of configurations. Intuitively, $\mathcal{T}$ and $\mathcal{P}$ respectively represent a minimal set of tasks and phasers that are required in any configuration denoted by the constraint. In addition:

$-6 v: \mathrm{B} \rightarrow \mathbb{B}^{\{*\}}$ and seq $: \mathcal{T} \rightarrow \operatorname{UnrSuff}{ }^{\{*\}}$ respectively represent, like for partial configurations, a valuation of the shared Boolean variables and a mapping of tasks to their control sequences.

- gap $: \mathcal{T} \rightarrow$ totalFunctions $(\mathcal{P}, \mathbb{G})$ constrains relations between $\mathcal{T}$-tasks and $\mathcal{P}$-phasers by associating to each task $t$ a mapping gap $(t)$ that defines for each phaser $p$ a named gap $($ var, val $) \in \mathbb{G}$ capturing the relation of $t$ and $p$.

- egap $: \mathcal{P} \rightarrow \mathbb{N}^{2}$ associates lower bounds (ew, es) on gaps of tasks that are registered to $\mathcal{P}$-phasers but which are not explicitly captured by $\mathcal{T}$. This is described further in the constraints denotations below.

We write isReg(gap,t,p) to mean the task $t$ is registered to the phaser $p$, i.e., $\operatorname{gap}(t)(p) \notin\left(\mathrm{V}^{\{-, *\}} \times\{\mathrm{nreg}\}\right)$. A constraint $\phi$ is said to be free (resp. $B$ gap-bounded or $B$-good) if the set $\mathcal{G}=\{\operatorname{gap}(t)(p) \mid t \in \mathcal{T}, p \in \mathcal{P}\}$ is free (resp. $B$-gap-bounded or $B$-good). The dimension of a constraint is the number of phasers it requires (i.e., $|\mathcal{P}|$ ). A set of constraints $\Phi$ is said to be free, $B$-gapbounded, $B$-good or $K$-dimension-bounded if each of its constraints are.

Denotations. We write $c \models \phi$ to mean constraint $\phi=\left(\mathcal{T}_{\phi}, \mathcal{P}_{\phi}, 6 v_{\phi}, s e q_{\phi}, g a p_{\phi}\right.$, $\left.\operatorname{egap}_{\phi}\right)$ denotes configuration $c=\left(\mathcal{T}_{c}, \mathcal{P}_{c}, b v_{c}\right.$, seq $_{c}$, phase $\left.{ }_{c}\right)$. Intuitively, the configuration $c$ should have at least as many tasks (captured by a surjection $\tau$ from a subset $\mathcal{T}_{c}^{1}$ of $\mathcal{T}_{c}$ to $\mathcal{T}_{\phi}$ ) and phasers (captured by a bijection $\pi$ from a subset $\mathcal{P}_{c}^{1}$ of $\mathcal{P}_{c}$ to $\left.\mathcal{P}_{\phi}\right)$. Constraints on the tasks and phasers in $\mathcal{T}_{c}^{1}$ and $\mathcal{P}_{c}^{1}$ ensure target configurations are reachable. Additional constraints on the tasks in $\mathcal{T}_{c}^{2}=\mathcal{T}_{c} \backslash \mathcal{T}_{c}^{1}$ ensure this reachability is not blocked by tasks not captured by $\mathcal{T}_{\phi}$. More formally:

1. for each $\mathrm{b} \in \mathrm{B},\left(6 v_{\phi}(\mathrm{b}) \neq 6 v_{c}(\mathrm{~b})\right) \Longrightarrow\left(6 v_{\phi}(\mathrm{b})=*\right)$, and

2. $\mathcal{T}_{c}$ and $\mathcal{P}_{c}$ can be written as $\mathcal{T}_{c}=\mathcal{T}_{c}^{1} \uplus \mathcal{T}_{c}^{2}$ and $\mathcal{P}_{c}=\mathcal{P}_{c}^{1} \uplus \mathcal{P}_{c}^{2}$, with

3. $\tau: \mathcal{T}_{c}^{1} \rightarrow \mathcal{T}_{\phi}$ is a surjection and $\pi: \mathcal{P}_{c}^{1} \rightarrow \mathcal{P}_{\phi}$ is a bijection, and

4. for $t_{c} \in \mathcal{T}_{c}^{1}$ with $t_{\phi}=\tau\left(t_{c}\right),\left(\operatorname{seq}_{\phi}\left(t_{\phi}\right) \neq \operatorname{seq}_{c}\left(t_{c}\right)\right) \Longrightarrow\left(s e q_{\phi}\left(t_{\phi}\right)=*\right)$, and

5. for each $p_{\phi}=\pi\left(p_{c}\right)$, there is a natural level $\ell: 0 \leq \ell$ such that:

(a) if $t_{c} \in \mathcal{T}_{c}^{1}$ with $t_{\phi}=\tau\left(t_{c}\right)$, phase ${ }_{c}\left(t_{c}\right)\left(p_{c}\right)=\left(\right.$ var $_{c}$, val $\left._{c}\right)$ and $\operatorname{gap}_{\phi}\left(t_{\phi}\right)\left(p_{\phi}\right)=$ $\left(v a r_{\phi}, v a l_{\phi}\right)$, then it is the case that:

i. $\left(v a r_{c} \neq v a r_{\phi}\right) \Longrightarrow\left(v a r_{\phi}=*\right)$, and 
ii. $\left(v a l_{c}=\mathrm{nreg}\right) \Longleftrightarrow\left(v a l_{\phi}=\mathrm{nreg}\right)$, and

iii. if $\left(v_{a l}=(w, s)\right)$ and $\left(v_{c a l}=(1 \mathrm{w}, 1 \mathrm{~s}, \mathrm{uw}, \mathrm{us})\right)$ then $1 \mathrm{w} \leq l-w \leq \mathrm{uw}$ and $1 \mathrm{~s} \leq s-\boldsymbol{l} \leq \mathrm{us}$.

(b) if $t_{c} \in \mathcal{T}_{c}^{2}$, then for each $p_{\phi}=\pi\left(p_{c}\right)$ with phase ${ }_{c}\left(t_{c}\right)\left(p_{c}\right)=\left(\operatorname{var}_{c},(w, s)\right)$ and $\operatorname{egap}\left(p_{\phi}\right)=($ ew, es $)$, we have: $($ es $\leq s-l)$ and $($ ew $\leq l-w)$

Intuitively, for each phaser, the bounds given by gap constrain the values of the phases belonging to tasks captured by $\mathcal{T}_{\phi}$ (i.e., those in $\mathcal{T}_{c}^{1}$ ) and registered to the given phaser. This is done with respect to some non-negative level, one per phaser. The same level is used to constrain phases of tasks registered to the phaser but not captured by $\mathcal{T}_{\phi}$ (i.e., those in $\mathcal{T}_{c}^{2}$ ). For these tasks, lower bounds are enough as we only want to ensure they do not block executions to target sets of configurations. We write $\llbracket \phi \rrbracket$ for $\{c \mid c \models \phi\}$.

Entailment. We write $\phi_{a} \sqsubseteq \phi_{b}$ to mean $\phi_{a}=\left(\mathcal{T}_{a}, \mathcal{P}_{a}, 6 v_{a}, \operatorname{seq}_{a}\right.$, gap $_{a}$, egap $\left._{a}\right)$ is entailed by $\phi_{b}=\left(\mathcal{T}_{b}, \mathcal{P}_{b}, 6 v_{b}, s e q_{b}, g a p_{b}, e g a p_{b}\right)$. This will ensure that configurations denoted by $\phi_{b}$ are also denoted by $\phi_{a}$. Intuitively, $\phi_{b}$ should have at least as many tasks (captured by a surjection $\tau$ from a subset $\mathcal{T}_{b}^{1}$ of $\mathcal{T}_{b}$ to $\mathcal{T}_{a}$ ) and phasers (captured by a bijection $\pi$ from a subset $\mathcal{P}_{b}^{1}$ of $\mathcal{P}_{b}$ to $\mathcal{P}_{a}$ ). Conditions on tasks and phasers in $\mathcal{T}_{b}^{1}$ and $\mathcal{P}_{b}^{1}$ ensure the conditions in $\phi_{a}$ are met. Additional conditions on the tasks in $\mathcal{T}_{b}^{2}=\mathcal{T}_{b} \backslash \mathcal{T}_{b}^{1}$ ensure at least the egap ${ }_{a}$ conditions in $\phi_{a}$ are met. More formally:

1. $\left(6 v_{a}(\mathrm{~b}) \neq 6 v_{b}(\mathrm{~b})\right) \Longrightarrow\left(6 v_{a}(\mathrm{~b})=*\right)$, for each $\mathrm{b} \in \mathrm{B}$ and

2. $\mathcal{T}_{b}$ and $\mathcal{P}_{b}$ can be written as $\mathcal{T}_{b}=\mathcal{T}_{b}^{1} \uplus \mathcal{T}_{b}^{2}$ and $\mathcal{P}_{b}=\mathcal{P}_{b}^{1} \uplus \mathcal{P}_{b}^{2}$ with

3. $\tau: \mathcal{T}_{b}^{1} \rightarrow \mathcal{T}_{a}$ is a surjection and $\pi: \mathcal{P}_{b}^{1} \rightarrow \mathcal{P}_{a}$ is a bijection, and

4. $\left(\operatorname{seq}_{b}\left(t_{b}\right) \neq \operatorname{seq}_{a}\left(t_{a}\right)\right) \Longrightarrow\left(\operatorname{seq}_{b}\left(t_{b}\right)=*\right)$ for each $t_{b} \in \mathcal{T}_{b}^{1}$ with $t_{a}=\tau\left(t_{b}\right)$, and 5. for each phaser $p_{a}=\pi\left(p_{b}\right)$ in $\mathcal{P}_{a}$ :

(a) if $\operatorname{egap}_{a}\left(p_{a}\right)=\left(\mathrm{ew}_{a}, \mathrm{es}_{a}\right)$ and $\operatorname{egap}_{b}\left(p_{b}\right)=\left(\mathrm{ew}_{b}, \mathrm{es}_{b}\right)$ then $\mathrm{ew}_{a} \leq \mathrm{ew}_{b}$ and $\mathrm{es}_{a} \leq \mathrm{es}_{b}$

(b) for each $t_{b} \in \mathcal{T}_{b}^{1}$ with $t_{a}=\tau\left(t_{b}\right)$ and $g_{a p}\left(t_{a}\right)\left(p_{a}\right)=\left(v_{a} r_{a}, v a l_{a}\right)$, and $\operatorname{gap}_{b}\left(t_{b}\right)\left(p_{b}\right)=\left(\operatorname{var}_{b}, v l_{b}\right)$, it is the case that:

i. $\left(\operatorname{var}_{b} \neq \operatorname{var}_{a}\right) \Longrightarrow\left(\operatorname{var}_{a}=*\right)$, and

ii. $\left(v a l_{b}=\mathrm{nreg}\right) \Longleftrightarrow\left(v a l_{a}=\mathrm{nreg}\right)$, and

iii. if $v a l_{a}=\left(1 \mathrm{w}_{a}, l \mathbf{s}_{a}, \mathrm{uw}_{a}, \mathrm{us}_{a}\right)$ and $v a l_{b}=\left(1 \mathrm{w}_{b}, 1 \mathbf{s}_{b}, \mathrm{uw}_{b}, \mathrm{us}_{b}\right)$, then $\left(1 \mathrm{w}_{a} \leq 1 \mathrm{w}_{b}\right),\left(\mathbf{l} \mathrm{s}_{a} \leq 1 \mathrm{~s}_{b}\right),\left(\mathrm{uw}_{b} \leq \mathrm{uw}_{a}\right)$ and $\left(\mathrm{us}_{b} \leq \mathrm{us}_{a}\right)$.

(c) for each $t_{b} \in \mathcal{T}_{b}^{2}$ with gap $\left.\operatorname{gat}_{b}\right)\left(p_{b}\right)=\left(\operatorname{var},\left(\mathbf{l} \mathrm{w}_{a}, \mathbf{l} \mathbf{s}_{a}\right.\right.$, uw $\left.\left._{a}, \mathrm{us}_{a}\right)\right)$, with $\operatorname{egap}_{a}\left(p_{a}\right)=\left(\mathrm{ew}_{a}, \mathrm{es}_{a}\right)$, both $\left(\mathrm{ew}_{a} \leq 1 \mathrm{w}_{b}\right)$ and $\left(\mathrm{es}_{a} \leq 1 \mathrm{~s}_{b}\right)$ hold.

The following lemma shows that it is safe to eliminate entailing constraints in the working list procedure of Sect. 5 .

\section{Lemma 2 (Constraint entailment). $\phi_{a} \sqsubseteq \phi_{b}$ implies $\llbracket \phi_{b} \rrbracket \subseteq \llbracket \phi_{a} \rrbracket$}

A central contribution that allows establishing the positive results of Sect. 5 is to show $\sqsubseteq$ is actually $\mathcal{W} \mathcal{Q O}$ on any $K$-dimension-bounded and $B$-good set of constraints. For this, we prove $\left(\mathcal{M}\left(\operatorname{UnrSuff} \times \mathcal{G}^{K}\right), \exists \preceq \forall\right)$ is $\mathcal{W} \mathcal{Q O}$ if $\mathcal{G}$ is $B$-good, where $\mathcal{M}\left(\operatorname{UnrSuff} \times \mathcal{G}^{K}\right)$ is the set of multisets over UnrSuff $\times \mathcal{G}^{K}$ 
and $M \exists \preceq \forall M^{\prime}$ requires each $\left(\mathbf{s}^{\prime}, g_{1}^{\prime}, \ldots, g_{K}^{\prime}\right) \in M^{\prime}$ may be mapped to some $\left(\mathbf{s}, g_{1}, \ldots, g_{K}\right) \in M$ for which $\mathbf{s}=\mathbf{s}^{\prime}$ and $g_{i} \unlhd g_{i}^{\prime}$ for each $i: 1 \leq i \leq K$ (written $\left.\left(\mathbf{s}, g_{1}, \ldots, g_{K}\right) \preceq\left(\mathbf{s}^{\prime}, g_{1}^{\prime}, \ldots, g_{K}^{\prime}\right)\right)$. Intuitively, we need to use $\exists \preceq_{\forall}$, and not simply $\forall \preceq_{\exists}$, in order to "cover all registered tasks" in the larger constraint as otherwise some tasks may block the path to the target configurations. Rado's structure [12,13] shows that, in general, $\left(\mathcal{M}(S), \exists_{\forall}\right)$ need not be $\mathcal{W} \mathcal{Q O}$ just because $\preceq$ is $\mathcal{W} \mathcal{Q O}$ over $S$. The proof details can be found in [10].

Theorem 3. $(\Phi, \sqsubseteq)$ is $\mathcal{W} \mathcal{Q O}$ if $\Phi$ is $K$-dimension-bounded and B-good for some pre-defined $K, B \in \mathbb{N}$.

\section{A Symbolic Verification Procedure}

We use the constraints defined in Sect. 4 as a symbolic representation in an instantiation of the classical framework of Well-Structured-Transition-Systems $[1,8]$. The instantiation (described in [10]) is a working-list procedure that takes as arguments a program prg and a $\sqsubseteq$-minimal set $\Phi$ of constraints denoting the targeted set of configurations. Such constraints can be easily built from the partial configurations described in Fig. 2.

The procedure computes a fixpoint using the entailment relation of Sect. 4 and a predecessor computation that results, for a constraint $\phi$ and a statement stmt, in a finite set pre stmt $=\left\{\phi^{\prime} \mid \phi \underset{\text { stmt }}{\longrightarrow} \phi^{\prime}\right\}$. Figure 4 describes part of the computation for the v.signal() instruction (see [10] for other instructions). For all but atomic statements, the set pre stmt $=\left\{\phi^{\prime} \mid \phi \underset{\text { stmt }}{\longrightarrow} \phi^{\prime}\right\}$ is exact in the sense that $\left\{c^{\prime} \mid c \in \llbracket \phi \rrbracket\right.$ and $\left.c^{\prime} \underset{\text { stmt }}{\longrightarrow} c\right\} \subseteq \bigcup_{\phi^{\prime} \in \text { pre }_{\text {stmt }}} \llbracket \phi^{\prime} \rrbracket \subseteq$ $\left\{c^{\prime} \mid c \in \llbracket \phi \rrbracket\right.$ and $\left.c^{\prime} \underset{\text { stmt }}{\longrightarrow} c\right\}$. Intuitively, the predecessors calculation for the atomic v.next ()$\{$ stmt $\}$ is only an over-approximation because such an instruction can encode a test-and-set operation. Our representation allows for more tasks, but the additional tasks may not be able to carry the atomic operation. We would therefore obtain a non-exact over-approximation and avoid this issue by only applying the procedure to non-atomic programs. We can show the following theorems.

$$
\begin{aligned}
& \mathbf{s}^{\prime}, \mathbf{s} \in \text { UnrSuff } \quad \mathbf{s}^{\prime}=\mathrm{v} \cdot \operatorname{signal}() ; \mathbf{s} \quad s e q^{\prime}=\operatorname{seq}\left[t \leftarrow \mathbf{s}^{\prime}\right] \quad g a p(t)(p)=\left(\mathrm{v},\left(1 \mathrm{w}_{t}, 1 \mathbf{s}_{t}, \mathrm{uw}_{t}, \mathrm{us}_{t}\right)\right.
\end{aligned}
$$

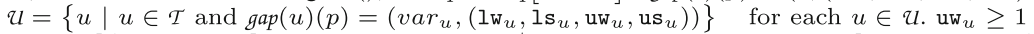

$$
\begin{aligned}
& \operatorname{gap}_{1}=\operatorname{gap}\left[\left\{u \leftarrow \operatorname{gap}(u)\left[p \leftarrow\left(\operatorname{var}_{u},\left(\left(1 \mathrm{w}_{u}-1\right)^{+}, \mathrm{ls}_{u}+1, \mathrm{uw}_{u}-1, \mathrm{us}_{u}+1\right)\right)\right] \mid u \in u \backslash\{t\}\right\}\right] \\
& \frac{\operatorname{gap}^{\prime}=\operatorname{gap}_{1}\left[t \leftarrow \operatorname{gap}_{1}\left[p \leftarrow\left(\operatorname{var}_{t},\left(\left(1 \mathrm{w}_{t}-1\right)^{+}, \mathrm{ls}_{t}, \mathrm{uw}_{t}-1, \mathrm{us}_{t}\right)\right)\right]\right] \quad \operatorname{egap}^{\prime}=\operatorname{egap}\left[p \leftarrow(\mathrm{ew}-1)^{+}, \mathrm{es}+1\right]}{(\mathcal{T}, \mathcal{P}, 6 v, \text { seq, gap }, \text { egap }) \underset{\mathrm{v} \cdot \mathrm{signal}()}{\longrightarrow}\left(\mathcal{T}^{\prime}, \mathcal{P}^{\prime}, 6 v, \operatorname{seq}^{\prime}, \operatorname{gap}^{\prime}, \operatorname{egap}^{\prime}\right)}
\end{aligned}
$$

Fig. 4. Part of the predecessors computation for the v.signal() phaser statement where $x^{+}$stands for $\max (0, x)$. Task $t \in \mathcal{T}$ is registered to $p \in \mathcal{P}$ and refers to it with $\mathrm{v}$ with all registered tasks having a non-zero upper bound on their waiting phases. 
Theorem 4. Control reachability is decidable for non-atomic phaser programs generating a finite number of phasers.

The idea is to systematically drop, in the instantiated backward procedure, constraints violating $K$-dimension-boundedness (as none of the denoted configurations is reachable). Also, the set of target constraints is free (since we are checking control reachability) and this is preserved by the predecessors compu-

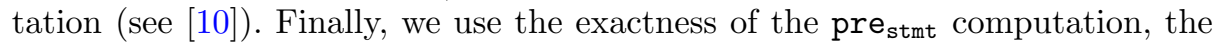
soundness of the entailment relation and Theorem 3 . We can use a similar reasoning for plain reachability of programs generating a finite number of phasers and bounded gap-values for each phaser.

Theorem 5. Plain reachability is decidable for non-atomic phaser programs generating a finite number of phasers with, for each phaser, bounded phase gaps.

\section{Limitations of Deciding Reachability}

Assume a program prg $=(\mathrm{B}, \mathrm{V}, \mathrm{T})$ and its initial configuration $c_{\text {init }}$. We show a number of parameterized reachability problems to be undecidable. First, we address checking control reachability when restricting to configurations with at most $K$ task-referenced phasers. We call this $K$-control-reachability.

Definition 3 ( $K$-control-reachability). Given a partial control configuration $c$, we write reach $\cos _{K}(\mathrm{prg}, c)$, and say $c$ is $K$-control-reachable, to mean there are $n+1$ configurations $\left(c_{i}\right)_{i: 0 \leq i \leq n}$, each with at most $K$ reachable phasers (i.e., phasers referenced by at least a task variable) s.t. $c_{\text {init }}=c_{0}$ and $c_{i} \longrightarrow c_{i+1}$ for $i: 0 \leq i<n-1$ with $c_{n}$ equivalent to a configuration that includes $c$.

Theorem 6. K-control-reachability is undecidable in general.

Proof sketch. Encode state reachability of an arbitrary Minsky machine with counters $x$ and $y$ using $K$-control-reachability of a suitable phaser program. The program (see [10]) has five tasks: main, xTask, yTask, child1 and child2. Machine states are captured with shared variables and counter values with phasers $\mathrm{xPh}$ for counter $x$ (resp. $\mathrm{yPh}$ for counter $y$ ). Then, (1) spawn an instance of xTask (resp. yTask) and register it to $\mathrm{xPh}$ (resp. $\mathrm{yPh}$ ) for increments, and (2) perform a wait on $\mathrm{xPh}$ (resp. $\mathrm{yPh}$ ) to test for zero. Decrementing a counter, say $x$, involves asking an $\mathrm{xTask}$, via shared variables, to exit (hence, to deregister from $\mathrm{xPh}$ ). However, more than one task might participate in the decrement operation. For this reason, each participating task builds a path from $\mathrm{xPh}$ to child2 with two phasers. If more than one xTask participates in the decrement, then the number of reachable phasers of an intermediary configuration will be at least five.

Theorem 7. Control reachability is undecidable if atomic statements are allowed even if only a finite number of phasers is generated. 
Proof sketch. Encode state reachability of an arbitrary Minsky machine with counters $x$ and $y$ using a phaser program with atomic statements. The phaser program (see [10]) has three tasks: main, xTask and yTask and encodes machine states with shared variables. The idea is to associate a phaser $\mathrm{xPh}$ to counter $x$ (resp. yPh to $y$ ) and to perform a signal followed by a wait on $\mathrm{xPh}$ (resp. $\mathrm{yPh}$ ) to test for zero. Incrementing and decrementing is performed by asking spawned tasks to spawn a new instance or to deregister. Atomic-next statements are used to ensure exactly one task is spawned or deregistered.

Finally, even with finite numbers of tasks and phasers, but with arbitrary gap-bounds, we can show [9] the following.

Theorem 8. Plain reachability is undecidable if generated gaps are not bounded even when restricting to non-atomic programs with finite numbers of phasers.

Table 1. Findings summary: ctrl stands for control reachability and plain for plain reachability; atomic stands for allowing the v.next()\{stmt\} atomic instruction and non-atomic for forbidding it (resulting in non-atomic programs). Decidable problems are marked with $\boldsymbol{\checkmark}$ and undecidable ones with $\boldsymbol{x}$.

\begin{tabular}{|c|c|c|c|c|}
\hline \multirow{3}{*}{$\begin{array}{c}\text { Bounded } \\
\text { gaps }\end{array}$} & \multicolumn{4}{|c|}{ Arbitrary numbers of tasks } \\
\hline & \multicolumn{2}{|c|}{ Finite dimension } & $K$-reachability & Arbitrary dimension \\
\hline & $\begin{array}{l}\text { ctrl atomic } \boldsymbol{x} \\
(\text { Theorem } 7)\end{array}$ & $\begin{array}{c}\text { plain non-atomic } \checkmark \\
(\text { Theorem } 5)\end{array}$ & \multirow{2}{*}{$\begin{array}{c}\text { ctrl non-atomic } \boldsymbol{x} \\
(\text { Theorem } 6)\end{array}$} & \multirow{2}{*}{$\begin{array}{l}\text { ctrl non-atomic } \boldsymbol{X} \\
(\text { Theorem 2) }\end{array}$} \\
\hline $\begin{array}{l}\text { Arbitrary } \\
\text { gaps }\end{array}$ & $\begin{array}{c}\text { ctrl non-atomic } \checkmark \\
(\text { Theorem } 4)\end{array}$ & $\begin{array}{c}\text { plain non-atomic } \boldsymbol{x} \\
\text { (From [9]) }\end{array}$ & & \\
\hline
\end{tabular}

\section{Conclusion}

We have studied parameterized plain (e.g., deadlocks) and control (e.g., assertions) reachability problems. We have proposed an exact verification procedure for non-atomic programs. We summarize our findings in Table 1. The procedure is guaranteed to terminate, even for programs that may generate arbitrary many tasks but finitely many phasers, when checking control reachability or when checking plain reachability with bounded gaps. These results were obtained using a non-trivial symbolic representation for which termination had required showing an $\exists \preceq \forall$ preorder on multisets on gaps on natural numbers to be a $\mathcal{W} \mathcal{Q O}$. We are working on a tool that implements the procedure to verify phaser programs that dynamically spawn tasks. We believe our general decidability results are useful to reason about synchronization constructs other than phasers. For instance, a traditional static barrier can be captured with one phaser and with bounded gaps (in fact one). Similarly, one phaser with one producer and arbitrary many consumers can capture futures where "gets" are modeled with waits. Also, test-and-set operations can model atomic instructions and may result in undecidability of reachability. This suggests more general applications of the work are to be investigated. 


\section{References}

1. Abdulla, P.A., Cerans, K., Jonsson, B., Tsay, Y.K.: General decidability theorems for infinite-state systems. In: Proceedings of the Eleventh Annual IEEE Symposium on Logic in Computer Science, LICS 1996, pp. 313-321. IEEE (1996)

2. Anderson, P., Chase, B., Mercer, E.: JPF verification of Habanero Java programs. SIGSOFT Softw. Eng. Notes 39(1), 1-7 (2014). https://doi.org/10.1145/2557833. 2560582. http://doi.acm.org/10.1145/2557833.2560582

3. de Boer, F.S., Clarke, D., Johnsen, E.B.: A complete guide to the future. In: De Nicola, R. (ed.) ESOP 2007. LNCS, vol. 4421, pp. 316-330. Springer, Heidelberg (2007). https://doi.org/10.1007/978-3-540-71316-6_22

4. Cavé, V., Zhao, J., Shirako, J., Sarkar, V.: Habanero-Java: the new adventures of old x10. In: Proceedings of the 9th International Conference on Principles and Practice of Programming in Java, pp. 51-61. ACM (2011)

5. Charles, P., et al.: X10: an object-oriented approach to non-uniform cluster computing. SIGPLAN Not. 40(10), 519-538 (2005). https://doi.org/10.1145/1103845. 1094852. http://doi.acm.org/10.1145/1103845.1094852

6. Cogumbreiro, T., Hu, R., Martins, F., Yoshida, N.: Dynamic deadlock verification for general barrier synchronisation. In: 20th ACM SIGPLAN Symposium on Principles and Practice of Parallel Programming, PPoPP 2015, pp. 150-160. ACM, New York (2015). https://doi.org/10.1145/2688500.2688519. http://doi.acm.org/ $10.1145 / 2688500.2688519$

7. Cogumbreiro, T., Shirako, J., Sarkar, V.: Formalization of Habanero phasers using Coq. J. Log. Algebraic Methods Program. 90, 50-60 (2017). https://doi.org/10. 1016/j.jlamp.2017.02.006. http://www.sciencedirect.com/science/article/pii/S23 52220816300839

8. Finkel, A., Schnoebelen, P.: Well-structured transition systems everywhere!. Theoret. Comput. Sci. 256(1), 63-92 (2001). https://doi.org/10.1016/S03043975(00)00102-X. http://www.sciencedirect.com/science/article/pii/S0304397 $50000102 \mathrm{X}$

9. Ganjei, Z., Rezine, A., Eles, P., Peng, Z.: Safety verification of phaser programs. In: Proceedings of the 17th Conference on Formal Methods in Computer-Aided Design, FMCAD 2017, pp. 68-75. FMCAD Inc., Austin (2017). http://dl.acm. org/citation.cfm?id=3168451.3168471

10. Ganjei, Z., Rezine, A., Henrio, L., Eles, P., Peng, Z.: On reachability in parameterized phaser programs. arXiv:1811.07142 (2019)

11. Havelund, K., Pressburger, T.: Model checking Java programs using Java pathfinder. Int. J. Softw. Tools Technol. Transfer (STTT) 2(4), 366-381 (2000)

12. Jancar, P.: A note on well quasi-orderings for powersets. Inf. Process. Lett. 72(5-6), 155-160 (1999). https://doi.org/10.1016/S0020-0190(99)00149-0

13. Marcone, A.: Fine analysis of the quasi-orderings on the power set. Order 18(4), 339-347 (2001). https://doi.org/10.1023/A:1013952225669

14. Minsky, M.L.: Computation: Finite and Infinite Machines. Prentice-Hall Inc., Englewood Cliffs (1967)

15. Shirako, J., Peixotto, D.M., Sarkar, V., Scherer, W.N.: Phasers: a unified deadlockfree construct for collective and point-to-point synchronization. In: 22nd Annual International Conference on Supercomputing, pp. 277-288. ACM (2008)

16. Valiant, L.G.: A bridging model for parallel computation. CACM 33(8), 103 (1990)

17. Willebeek-LeMair, M.H., Reeves, A.P.: Strategies for dynamic load balancing on highly parallel computers. IEEE Trans. Parallel Distrib. Syst. 4(9), 979-993 (1993) 
Open Access This chapter is licensed under the terms of the Creative Commons Attribution 4.0 International License (http://creativecommons.org/licenses/by/4.0/), which permits use, sharing, adaptation, distribution and reproduction in any medium or format, as long as you give appropriate credit to the original author(s) and the source, provide a link to the Creative Commons license and indicate if changes were made.

The images or other third party material in this chapter are included in the chapter's Creative Commons license, unless indicated otherwise in a credit line to the material. If material is not included in the chapter's Creative Commons license and your intended use is not permitted by statutory regulation or exceeds the permitted use, you will need to obtain permission directly from the copyright holder. 